\title{
Multi-dimensional fuzzy transform and projection
}

\author{
Jean-François Crouzet \\ Institut Montpelliérain Alexander Grothendieck \\ Université de Montpellier \\ Place E. Bataillon, 34095 Montpellier cedex 5, France \\ Tel.: +334671445 21; fax: +334671435 58. \\ E-mail address: jean-francois.crouzet@univ-montp2.fr
}

\begin{abstract}
Fuzzy and inverse fuzzy transforms, introduced by I. Perfilieva, is an important tool for signal and image fuzzy representation. It led to several variants, as the natural least-squares minimization named fuzzy projection. We deal with this transform, proposing first to show its simplicity and its easy numerical implementation in any dimension of space. Secondly, we point out that a key parameter permits to control its numerical robustness. Thirdly, we show that this parameter also governs the stability of the representation by fuzzy projection, when choosing different partitions of the space. We conclude in discussing linear representation; especially triangular representation for signals and pyramidal representation for images.
\end{abstract}

Keywords: Fuzzy transform, fuzzy projection, signal representation, image representation, stability, robustness.

\section{Introduction}

Fuzzy and inverse fuzzy transforms for the representation of signal and images have been initiated by Irina Perfilieva in 2004 ([? ],[? ]) and has led to many applications in their processing ([? ], [? ], [? ], [? ], [? ], [? ], [? ]). In their continuity the author of the present study introduced in 2010 the operator of fuzzy projection for signals ([? ],[? ]). Independently, Giuseppe Patanè ([? ]) proposed in 2011 a study of the fuzzy transform via leastsquares approximation that brought out the same operator. Fuzzy projection (F-projection) proved to perform a good interpolation of the samples of a 
signal obtained by fuzzy transform (F-transform). In particularly it handles correctly high frequencies, even in presence of an important noise ([?]).

In this paper, we present and discuss of F-projection in higher dimension of space. We propose to show it is a simple tool, very easy to implement numerically, especially for image analysis. (We will deal in a future work ([?]) with numerical experiments such as compression, denoising and restauration of images, particularly a comparison with wavelet representation.)

Moreover, we point out that a key parameter permits to control the robustness of fuzzy projection, i.e. how much it is numerically well conditioned. It also indicates its stability, say its sensitivity, when choosing different partitions of the space, i.e. different generic kernels of the fuzzy partition: the effect of this choice does not modify significantly the representation process. This parameter is simply the squared $L^{2}$-norm of the generic kernel $\mu$, generating the fuzzy partition in dimension 1: $a=\|\mu\|_{2}^{2}$. As it measures, in any dimension of space, how the generic kernel of the fuzzy partition is concentrated around the center of its supporting cell, we name it the 'localization parameter'. Thanks to the parameter $a$, we discuss the linear representation of the considered object. More precisely, we suggest that choosing a linear fuzzy partition (a triangular one for signals, or a pyramidal one for images) is a reasonable compromise between numerical robustness, stability of the representation, and the requirements of a fuzzy representation of a signal or a multi-dimensional object.

In section 2, we recall definitions and results necessary to present F-projection in dimension 1 of space that are been presented and proved in [? ]. In section 3, we highlight the natural analogy of these results in dimension 2 of space. In section 4, a rapid generalization is made in any dimension. Section 5 adresses a notion of stability for this representation, highlighting it is controlled by the 'localisation parameter'. We conclude with discussing the relevance of a linear fuzzy partition.

\section{Fuzzy projection in dimension 1}

Following the seminal work of Irina Perfilieva ([? ],[? ]) on direct and inverse F-transform, we collect in this section the results on F-projection that were proved in [?] and [? ]. This will permit to introduce easily multidimensional F-projection, and the role of the 'localization parameter'. 


\subsection{Uniform fuzzy partition}

A fuzzy partition $\mathcal{P}=\left\{\mu_{k}, k=0 \ldots p+1\right\}$ of an interval is defined as follows.

Definition 2.1.1. (fuzzy partition $\mathcal{P}$ )

Let $\mathcal{S}=\left\{\omega_{k}=k \Delta, k=0 \ldots p+1\right\}$ be an uniform subdivision of $[0, d]$ with diameter $\Delta=d /(p+1)$. Let $\mu \in \mathcal{C}^{0}([-1,1])$, the generic kernel of the partition, be an even continuous function such that $\mu(0)=1, \mu(1)=0$ and $\mu(1-x)+\mu(x)=1, \forall x \in[-1,1]$. Kernels $\mu_{k}$ of the partition $\mathcal{P}$ are defined by $\mu_{k}(x)=\mu\left(\frac{x-\omega_{k}}{\Delta}\right)$, and have thus for support the cells $\Omega_{k}=\left[\omega_{k-1}, \omega_{k+1}\right]$ (with the convention that $\omega_{-1}=\omega_{0}=0$ and $\omega_{p+2}=\omega_{p+1}=d$.)

Then, it is immediate to check that $\mathcal{P}$ is a partition of unity on $[0, d]$ :

Property 2.1.1. (partition of unity)

i)

$$
\forall x \in[0, d], \sum_{k=0}^{p+1} \mu_{k}(x)=1
$$

ii)

$$
\forall k=0 \ldots p+1, \frac{1}{\Delta} \int_{0}^{d} \mu_{k}=\frac{1}{\Delta} \int_{\Omega_{k}} \mu_{k}=\int_{-1}^{1} \mu=1
$$

\subsection{Fuzzy transform}

Denote $L^{2}([0, d])$ the space of finite energy signals with norm $\|f\|_{2}=$ $\left(\int_{0}^{d} f^{2}\right)^{1 / 2}$ and scalar product $(f \mid g)=\int_{0}^{d} f g$. Defined by Irina Perfilieva ([? ],[? ]) on the subspace $\mathcal{C}^{0}([0, d])$, the more general fuzzy transform we consider in this study is the operator $F: L^{2}([0, d]): \mapsto \mathbb{R}^{p+2}$ such that:

Definition 2.2.1. (fuzzy transform F: sampling)

$$
\forall k=0 \ldots p+1,(F f)_{k}:=\frac{\int_{0}^{d} f \mu_{k}}{\int_{0}^{d} \mu_{k}}=\frac{1}{\Delta}\left(f \mid \mu_{k}\right)
$$

Fuzzy transform thus consists in taking a sequence of means, weighted by kernels $\mu_{k}$, of the signal $f$. Note that it maps constant functions to a constant sampling with the same value. We also need to express its adjoint. Consider, for $u=\left(u_{0}, \ldots, u_{p+1}\right)$ and $v=\left(v_{0}, \ldots, v_{p+1}\right)$, the scalar 
product in $\mathbb{R}^{p+2}: \quad u \cdot v=\Delta \sum_{k=0}^{p+1} u_{k} v_{k}$. For $\gamma=\left(\gamma_{0}, \ldots, \gamma_{p+1}\right), \gamma \cdot F f=$ $\Delta \sum_{k=0}^{p+1} \gamma_{k}(F f)_{k}=\sum_{k=0}^{p+1} \gamma_{k}\left(\mu_{k} \mid f\right)=\left(f \mid \sum_{k=0}^{p+1} \gamma_{k} \mu_{k}\right)$ yields the expression of $F^{*}: \mathbb{R}^{p+2} \mapsto L^{2}([0, d])$ :

Proposition 2.2.1. (adjoint fuzzy transform $F^{*}$ : interpolation)

For $\gamma=\left(\gamma_{0}, \ldots, \gamma_{p+1}\right)$, the adjoint fuzzy transform is

$$
F^{*} \gamma=\sum_{k=0}^{p+1} \gamma_{k} \mu_{k}
$$

and can be viewed as an interpolation of the finite sequence $\gamma_{0}, \ldots, \gamma_{p+1}$ by kernels $\mu_{k}$ of the partition.

Remark 2.2.1. By 'interpolation' we mean in this study the passage from a discrete sampled representation of a signal (say $F f$ in our context) to a continuous representation by mean of kernels functions $\mu_{k}$ of the partition (say for instance $F^{*} F f$ for the inverse fuzzy transform introduced by I. Perfilieva). It is directly related to fuzzy projection that will be treated in the following section, where the interpolation functions are different.

\subsection{Fuzzy projection}

Let us first define the $p+2$-dimensional sub-space $\Pi$ of functions generated by partition $\mathcal{P}$, or, equivalently, the image of $F^{*}$ :

Definition 2.3.1. (projection sub-space П)

$$
\Pi=\left\{\sum_{k=0}^{p+1} \gamma_{k} \mu_{k}, \gamma \in \mathbb{R}^{p+2}\right\}
$$

For $f \in L^{2}([0, d])$, F-projection is defined by:

Definition 2.3.2. (F-projection $P$ )

$$
P f=\operatorname{Argmin}_{g \in \Pi}\|f-g\|_{2}
$$

Computing $P f$ is thus equivalent to find

$$
\alpha=\operatorname{Argmin}_{\gamma \in \mathbb{R}^{p+2}}\left\|f-F^{*} \gamma\right\|_{2}
$$

Its solution is given by the normal equation $F F^{*} \alpha=F f$, so that F-projection is the the classical pseudo-inverse projector $([?])$ : 
Proposition 2.3.1. (pseudo-inverse)

The expression of the F-projection $P: L^{2}([0, d]) \mapsto \Pi$ is the following:

$$
P f=F^{*}\left(F F^{*}\right)^{-1} F f
$$

It is established in [? ] uniform and quadratic convergence (when $p \rightarrow \infty$, i.e. $\left.\Delta=\frac{d}{p+1} \rightarrow 0\right)$ :

Theorem 2.3.1. (convergence)

i) Let $f \in \mathcal{C}^{0}([0, d])$. Then $\forall \varepsilon>0, \exists \Delta \mid\|f-P f\|_{\infty} \leq \varepsilon$.

ii) Let $f \in L^{2}([0, d])$. Then $\forall \varepsilon>0, \exists \Delta \mid\|f-P f\|_{2} \leq \varepsilon$.

Let us now define the announced control parameter with which we will deal till the end of the present study.

Definition and Property 2.3.1. (localization parameter a)

We define

$$
a=\|\mu\|_{2}^{2}=\int_{-1}^{1} \mu^{2}
$$

as the 'localization parameter' for $\mu$. The fact that $a=1 / 2+4 \int_{0}^{1 / 2}(\mu-1 / 2)^{2}$ shows that it represents the degree of localization of $\mu$ in the central interval $[-1 / 2,1 / 2]$ of its support $[-1,1]$. It varies from $1 / 2$ (limit case of the step function with maximum overlap, $\mu=\frac{1}{2} \chi_{[-1,1]}$ ) to 1 (limit case of the basic step function with no overlap, $\left.\mu=\chi_{[-1 / 2,1 / 2]}\right)$ :

$$
\frac{1}{2}<a<1
$$

Remark 2.3.1. There exists, of course, an infinity of generic kernels providing a same parameter a. For instance, the triangle kernel, corresponding to linear fuzzy sampling, verifies $a=2 / 3$, and it is easy to construct other ones with the same parameter a, but presenting oscillating shapes (even if they strictly decrease on $[0,1]$, as demanded). Nevertheless, it is reasonable to keep in view this most natural and simple kernel.

Let us now identify $F F^{*}$ to its matrix $M$ in the canonical basis of $\mathbb{R}^{p+2}$. Then, as proved in [?], we can state the following result: 
Theorem 2.3.2. (expression of F-projection and role of a)

i) $F F^{*}$ corresponds to $a(p+2) \times(p+2)$ (almost bistochastic) symmetrical tridiagonal matrix

$$
M=\left[\begin{array}{ccccccc}
a & \frac{1-a}{2} & & & & & \\
\frac{1-a}{2} & a & \frac{1-a}{2} & & & (0) & \\
& \frac{1-a}{2} & a & \frac{1-a}{2} & & & \\
& & \cdot & \cdot & \cdot & & \\
& (0) & & & \frac{1-a}{2} & a & \frac{1-a}{2} \\
& & & & & \frac{1-a}{2} & a
\end{array}\right]
$$

depending only on the localization parameter $a$.

ii) $M$ is invertible and has $p+2$ simple eigenvalues

$$
\lambda_{k}=a+(1-a) \cos \frac{k \pi}{p+1}
$$

verifying $0<2 a-1 \leq \lambda_{k} \leq 1, k=0 \ldots p+1$.

iii) The associated normalized eigenvectors are denoted $v_{k}$ and defined by

$$
\left(v_{k}\right)_{j}=\left(\frac{2}{d}\right)^{\frac{1}{2}} \cos \left(\frac{j k \pi}{p+1}\right)
$$

They do not depend on the generic kernel.

iv) The set of functions

$$
\mathcal{B}=\left\{f_{k}=\frac{1}{\sqrt{\lambda_{k}}} F^{*} v_{k}, k=0 \ldots p+1\right\}
$$

forms an orthonormal basis of the projection subspace $\Pi$. Thus, for any $f \in L^{2}([0, d])$ we have

$$
P f=\sum_{k=0}^{p+1}\left(f \mid f_{k}\right) f_{k}
$$

v) The functions

$$
\nu_{k}(x)=\sum_{j=0}^{p+1} M_{j, k}^{-1} \mu_{j}(x)
$$


make $P f$ reads equivalently as the following interpolation - natural continuous representation - of the fuzzy transform $F f$ :

$$
P f=\sum_{k=0}^{p+1}(F f)_{k} \nu_{k}
$$

\section{Remark 2.3.2.}

1) The first point of the theorem comes from a computation that the reader is invited to read in [? ]. Points ii) and ii) are then a classical property of tridiagonal matrixes (see for instance [? ]). As FF* is identified to the matrix $M$ with eigenvalues $\lambda_{k}$, point iv) is immediate.

2) The length $d$ of the interval $[0, d]$ where signals $f$ are considered appears in the formula $\left(v_{k}\right)_{j}=\left(\frac{2}{d}\right)^{\frac{1}{2}} \cos \left(\frac{j k \pi}{p+1}\right)$. This is due to the normalization of those vectors with the weighted scalar product u.v $=\Delta \sum_{k=0}^{p+1} u_{k} v_{k}$, where the diameter of the subdivision verifies $\Delta=d /(p+1)$.

3) Basis functions $f_{k}=\frac{1}{\sqrt{\lambda_{k}}} F^{*} v_{k}$ are the interpolations by $F^{*}$ of the finite sequence defined by $\left(v_{k}\right)_{j}=\left(\frac{2}{d}\right)^{\frac{1}{2}} \cos \left(\frac{j k \pi}{p+1}\right), j=0 \ldots p+1$. Thus, they look like a Fourier basis, but with the shape of the generic kernel $\mu$ appearing in $F^{*}$ (for instance a piecewise linear sinus-like function for linear interpolation with a triangular kernel.)

4) $\operatorname{Pf}(x)=F^{*} M^{-1} F f(x)=\sum_{j=0}^{p+1} \mu_{j}(x) \sum_{k=0}^{p+1} M_{j, k}^{-1}(F f)_{k}$ provides formula (??) in point $v)$.

Two important numerical requirements are that matrix $M$ must be well conditioned and that functions $\left\{\nu_{k}\right\}$ (that bring the discrete samples to a continuous interpolation representation) have well localized supports: that is what we call the 'robustness' of the fuzzy representation.

Theorem 2.3.3. (numerical robustness and role of a)

i)

$$
c:=\operatorname{cond}_{2}(M)=\frac{\max \left|\lambda_{k}\right|}{\min \left|\lambda_{k}\right|}=\frac{1}{2 a-1}
$$

ii) Each function $\nu_{k}(x)=\sum_{j=0}^{p+1} \mu_{j}(x) M_{j, k}^{-1}$, that operates the interpolation from the discrete sampling $F f$ to its continuous approximation $P f$, is exponentially decreasing in the sense that $\left|M_{j, k}^{-1}\right| \leq\left|M_{k, k}^{-1}\right| r^{|k-j|}$ for all indexes $j$ 
and $k$. The speed of decrease around the diagonal $0<r<1$ is given by

$$
r=\frac{1-a}{3 a-1}
$$

\section{Remark 2.3.3.}

1) The fact that that $\left|M_{j, k}^{-1}\right| \leq\left|M_{k, k}^{-1}\right| r^{|k-j|}$, for all indexes $j$ and $k$, means that the coefficient of the bounded and compactly supported kernels functions $\mu_{j}$ in the expression of $\nu_{k}$ are exponentially decreasing. Thus, function $\nu_{k}$ itself is also exponentially decreasing. An illustration is given in Figure?? for triangular kernels.

2) In the limit case of $\mu=\frac{1}{2} \chi_{[-1,1]}$, we have $a=1 / 2$, so that $c=\infty$ and $r=1$ : this is the worst numerical situation.

3) In the limit case of $\mu=\chi_{[-1 / 2,1 / 2]}$, we have $a=1$, so that $c=1$ and $r=0$ : this is the best numerical situation, but we will discuss this case in Section ??.

4) In the case of the classical triangular kernel $\mu=\operatorname{Tr} i_{[-1,1]}$, we have $a=2 / 3$, so that $c=3$ and $r=1 / 3$ : this is a good numerical situation; we will also discuss this case in the last Section ??, as it is the situation we want to bring out in this study.

5) In the case of the classical sinusoidal kernel: $\mu(x)=\frac{1}{2}(1+\cos (\pi x))$, we have $a=3 / 4$, so that $c=2$ and $r=1 / 5$ : this is an even better numerical situation we will also comment Section ??.

Thus, while $r=r(a)$ controls the localization of the interpolating functions, the condition number $c=c(a)$ controls the numerical sensibility of the representation. This shows that the localization parameter $a$ plays then a key role in basic numerical requirements. Moreover, we can also note that $a$ governs the rapidity of the uniform convergence in Theorem ??, as shown by its proof in [?].

\section{Fuzzy projection in dimension 2}

This section is very analogous to the previous one. However, as it is useful for image representation, we present all the results, following exactly the same way. Most of their proofs are let to the reader, as they are nearly the same that those in dimension 1 (presented in [?]). 


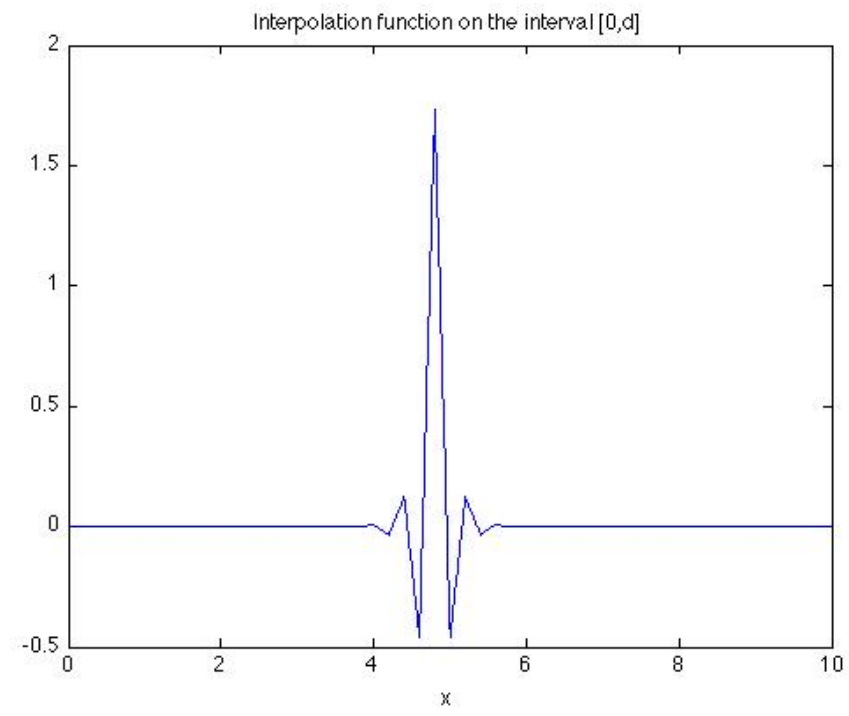

Figure 1: Example of function $\nu_{k}$ for a triangular generic kernel $\mu=\operatorname{Tri}_{[-1,1]}(d=10)$

\subsection{Uniform $2 D$ fuzzy partition}

Let us define a $2 D$ fuzzy partition $\mathcal{Q}=\left\{\mathcal{M}_{k, l},(k, l) \in[0 \ldots p+1]^{2}\right\}$ of the square in $[0, d]^{2}$ :

Definition 3.1.1. (2D fuzzy partition $\mathcal{Q})$

Let $\mathcal{T}=\left\{\left(\omega_{k}, \omega_{l}\right)=(k \Delta, l \Delta),(k, l) \in[0 \ldots p+1]^{2}\right\}$ be an uniform subdivision of $[0, d]^{2}$. Still, $\Delta=d /(p+1)$ is called the diameter of the subdivision. Recall that $\mu$ denotes the generic kernel in the $1 D$ Definition ??. Kernels $\mathcal{M}_{k, l}$ of the partition $\mathcal{T}$ are then defined by $\mathcal{M}_{k, l}(x, y)=$ $\mu_{k}(x) \mu_{l}(y):=\mu\left(\frac{x-\omega_{k}}{\Delta}\right) \mu\left(\frac{y-\omega_{l}}{\Delta}\right)$, and are supported in the cells $\Omega_{k, l}=$ $\left[\omega_{k-1}, \omega_{k+1}\right] \times\left[\omega_{l-1}, \omega_{l+1}\right]$. The generic kernel is $\mathcal{M}(x, y)=\mu(x) \mu(y)$, supported in the unit square $[-1,1]^{2}$.

Again, it is easy to verify that $\mathcal{Q}$ is a partition of unity on $[0, d]^{2}$, precisely:

Property 3.1.1. (2D partition of unity)

i)

$$
\forall(x, y) \in[0, d]^{2}, \sum_{k=0}^{p+1} \sum_{l=0}^{p+1} \mathcal{M}_{k, l}(x, y)=1
$$


ii)

$$
\forall(k, l) \in[0 \ldots p+1]^{2}, \frac{1}{\Delta^{2}} \iint_{[0, d]^{2}} \mathcal{M}_{k, l}=\frac{1}{\Delta^{2}} \iint_{\Omega_{k, l}} \mathcal{M}_{k, l}=\left(\int_{-1}^{1} \mu\right)^{2}=1
$$

Remark 3.1.1. As an illustration, the classical $1 D$ triangular generic kernels yields a partition with square pyramids.

\section{2. $2 D$ F-transform}

The $2 D$ fuzzy transform $F: L^{2}\left([0, d]^{2}\right) \mapsto \mathbb{R}^{p+2} \times \mathbb{R}^{p+2}$ is defined as follows:

Definition 3.2.1. (2D F-transform $F$ )

$$
\forall(k, l) \in[0 \ldots p+1]^{2},(F f)_{k, l}:=\frac{\iiint_{[0, d]^{2}} f \mathcal{M}_{k, l}}{\iint_{[0, d]^{2}} \mathcal{M}_{k, l}}=\frac{1}{\Delta^{2}}\left(f \mid \mathcal{M}_{k, l}\right)
$$

Considering on $\mathbb{R}^{p+2} \times \mathbb{R}^{p+2}$ the scalar product $u . v=$ $\Delta^{2} \sum_{k=0}^{p+1} \sum_{l=0}^{p+1} u_{k, l} v_{k, l}$, we have similarly the adjoint fuzzy transform $F^{*}: \mathbb{R}^{p+2} \times \mathbb{R}^{p+2} \mapsto L^{2}\left([0, d]^{2}\right)$ :

Proposition 3.2.1. (2D adjoint F-transform $F^{*}$ )

$$
F^{*} \gamma=\sum_{k=0}^{p+1} \sum_{l=0}^{p+1} \gamma_{k, l} \mathcal{M}_{k, l}
$$

\section{3. $2 D$ fuzzy projection}

The $(p+2)^{2}$-dimensional sub-space $\Pi$ generated by the partition $\mathcal{Q}$ is now:

Definition 3.3.1. (projection sub-space $\Pi$ )

$$
\Pi=\left\{\sum_{k=0}^{p+1} \sum_{l=0}^{p+1} \gamma_{k, l} \mathcal{M}_{k, l}, \gamma \in \mathbb{R}^{p+2} \times \mathbb{R}^{p+2}\right\}
$$

This leads to the same definition, expression and convergence results for F-projection: 
Definition 3.3.2. (2D F-projection P)

$$
P f=\operatorname{Argmin}_{g \in \Pi}\|f-g\|_{2}
$$

Proposition 3.3.1. (pseudo-inverse)

The expression of the $2 D$ F-projection $P: L^{2}\left([0, d]^{2}\right) \mapsto \Pi$ is the following:

$$
P f=F^{*}\left(F F^{*}\right)^{-1} F f
$$

Theorem 3.3.1. ( $2 D$ convergence)

i) Let $f \in \mathcal{C}^{0}\left([0, d]^{2}\right)$. Then $\forall \varepsilon>0, \exists \Delta \mid\|f-P f\|_{\infty} \leq \varepsilon$.

ii) Let $f \in L^{2}\left([0, d]^{2}\right)$. Then $\forall \varepsilon>0, \exists \Delta \mid\|f-P f\|_{2} \leq \varepsilon$.

Let us now identify $\mathbb{R}^{p+2} \times \mathbb{R}^{p+2}$ to $\mathbb{R}^{(p+2)^{2}}$ by the bijective map $(k, l) \mapsto$ $k+l(p+2)$. Then, the components of the fuzzy transform $(F f)_{k, l}$ will be stored in a $(p+2)^{2}$-dimensional vector. Thus, operator $F F^{*}$ can be identified to a matrix $N$ in the canonical basis of $\mathbb{R}^{(p+2)^{2}}$. This allows to get the following result. (Notations are the same as in $1 D$ Theorem ??; in particular for $a, \lambda_{k}, v_{k}$ and matrix $M$, given in formula (??) in the $1 D$ case.)

Theorem 3.3.2. (expression of

F-projection and role of a)

i) $F F^{*}$ corresponds to a $(p+2)^{2} \times(p+2)^{2}$ (almost bistochastic) matrix which is symmetrical block tridiagonal with symmetrical tridiagonal blocks:

$$
N=\left[\begin{array}{ccccccc}
a M & \frac{1-a}{2} M & & & & & \\
\frac{1-a}{2} M & a M & \frac{1-a}{2} M & & & (0) & \\
& \frac{1-a}{2} M & a M & \frac{1-a}{2} M & & & \\
& & \cdot & \cdot & \cdot & & \\
& (0) & & \cdot & \frac{1-a}{2} M & a M & \frac{1-a}{2} M \\
& & & & & \frac{1-a}{2} M & a M
\end{array}\right]
$$

depending only on the localization parameter a. 
ii) $F F^{*}$ is invertible and has $(p+2)^{2}$ simple eigenvalues

$$
\Lambda_{k, l}(a)=\lambda_{k}(a) \lambda_{l}(a)=\left(a+(1-a) \cos \frac{k \pi}{p+1}\right)\left(a+(1-a) \cos \frac{l \pi}{p+1}\right)
$$

verifying $0<(2 a-1)^{2} \leq \Lambda_{k, l} \leq 1, \forall(k, l) \in[0 \ldots(p+1)]^{2}$.

iii) The associated normalized 'eigenvectors' are $V_{k, l}=v_{k} \circ v_{l}$ with:

$$
\left(V_{k, l}\right)_{j, m}=\left(v_{k} \circ v_{l}\right)_{j, m}:=\left(v_{k}\right)_{j}\left(v_{l}\right)_{m}=\frac{2}{d} \cos \left(\frac{j k \pi}{p+1}\right) \cos \left(\frac{m l \pi}{p+1}\right)
$$

Again, they do not depend on the generic kernel.

iv) The set of functions

$$
\mathcal{B}=\left\{f_{k, l}=\frac{1}{\sqrt{\Lambda_{k, l}}} F^{*} V_{k, l},(k, l) \in[0 \ldots p+1]^{2}\right\}
$$

forms an orthonormal basis of the projection subspace П. Thus,

$$
P f=\sum_{k=0}^{p+1} \sum_{l=0}^{p+1}\left(f \mid f_{k, l}\right) f_{k, l}
$$

v) The functions

$$
\mathcal{N}_{k, l}(x, y)=\sum_{i=0}^{p+1} \sum_{j=0}^{p+1}\left(F F^{*}\right)_{(i, j),(k, l)}^{-1} \mathcal{M}_{i, j}(x, y)
$$

make $P f$ reads as the following interpolation of the fuzzy transform $F f$ :

$$
\operatorname{Pf}(x, y)=\sum_{k=0}^{p+1} \sum_{l=0}^{p+1}(F f)_{k, l} \mathcal{N}_{k, l}(x, y)
$$

Basic numerical requirements are the same: operator $F F^{*}$ (associated, for numerical purposes, to the matrix $N$ ) must be well conditioned and interpolation functions $\mathcal{N}_{k, l}$ well localized. Here is a slightly similar result, point ii) being however more technical. 
Theorem 3.3.3. ( $2 D$ numerical robustness and role of a)

i)

$$
C:=\operatorname{cond}_{2}\left(F F^{*}\right)=\frac{\max \left|\Lambda_{k, l}(a)\right|}{\min \left|\Lambda_{k, l}(a)\right|}=\frac{1}{(2 a-1)^{2}}
$$

ii) Interpolating function, $\mathcal{N}_{k, l}(x, y)=\sum_{i=0}^{p+1} \sum_{j=0}^{p+1}\left(F F^{*}\right)_{(i, j),(k, l)}^{-1} \mathcal{M}_{i, j}(x, y)$ are exponentially decreasing in the sense that, given the matrix $N$, there exists a constant $\alpha>0$ such that:

a) $\left|N_{i, j}^{-1}\right| \leq \alpha R^{i}$, if $i>j$ and $i$ is large enough,

b) $\left|N_{i, j}^{-1}\right| \leq \alpha R^{j}$, if $j>i$ and $j$ is large enough.

The speed of decrease away from the diagonal $0<R<1$ is given by

$$
R=\frac{1-a}{a+\sqrt{2 a-1}}
$$

\section{Proof of ii).}

It is proved in [? ] that a block tridiagonal block toeplitz matrix of the form

$$
T=\left[\begin{array}{ccccc}
A & B & & & \\
B & A & B & & (0) \\
& . & \cdot & . & \\
& (0) & \cdot & . & B \\
& & & B & A
\end{array}\right]
$$

with the same dimensions as $N$ verifies

a) $\left|T_{i, j}^{-1}\right| \leq \alpha\left|\rho_{p+1}\right|^{-i}$, if $i>j$ and $i$ is large enough,

b) $\left|T_{i, j}^{-1}\right| \leq \alpha\left|\rho_{p+1}\right|^{-j}$, if $j>i$ and $j$ is large enough,

where $\left\{\rho_{k}\right\}$ are the $2(p+1)$ eigenvalues, sorted in absolute value increasing order, of the $2(p+1) \times 2(p+1)$ matrix

$$
U=\left[\begin{array}{cc}
0 & B \\
-B^{-1} & B^{-1} A
\end{array}\right]
$$

In our case, we have (Theorem ??) $A=a M$ and $B=\frac{1-a}{2} M$. Denote $\lambda_{k}(A)$ and $\lambda_{k}(B)$ the respective eigenvalues of $A$ and $B$, and recall that $v_{k}$ are their corresponding common eigenvectors. When searching for eigenvectors of the form $V_{k}=\left[\begin{array}{c}\alpha_{k} v_{k} \\ v_{k}\end{array}\right]$ we have that $U V_{k}=\left[\begin{array}{cc}0 & B \\ -B^{-1} & B^{-1} A\end{array}\right]\left[\begin{array}{c}\alpha_{k} v_{k} \\ v_{k}\end{array}\right]=\left[\begin{array}{c}\lambda_{k}(B) v_{k} \\ \left(-\frac{\alpha_{k}}{\lambda_{k}(B)}+\frac{\lambda_{k}(A)}{\lambda_{k}(B)}\right) v_{k}\end{array}\right]$. A necessary 
condition for $V_{k}$ to be an eigenvector is then that $\alpha_{k}=\lambda_{k}(B) /\left(\frac{\lambda_{k}(A)-\alpha_{k}}{\lambda_{k}(B)}\right)$. This yields a second degree equation for $\alpha_{k}$ whose $2(p+1)$ solutions are, for $k=0 \ldots p+1$ :

- $\alpha_{k}^{(1)}=\frac{1}{2}\left(\lambda_{k}(A)+\sqrt{\lambda_{k}^{2}(A)-4 \lambda_{k}^{2}(B)}\right)$

- $\alpha_{k}^{(2)}=\frac{1}{2}\left(\lambda_{k}(A)-\sqrt{\lambda_{k}^{2}(A)-4 \lambda_{k}^{2}(B)}\right)$

Then, as $\rho_{k}$, the $2(p+1)$ eigenvalues of $U$, verify by definition $U V_{k}=U\left[\begin{array}{c}\alpha_{k} v_{k} \\ v_{k}\end{array}\right]=\rho_{k}\left[\begin{array}{c}\alpha_{k} v_{k} \\ v_{k}\end{array}\right]$, we have that $\rho_{k} \alpha_{k}=\lambda_{k}(B)$. It follows that, sorted in absolute value increasing order, they write, for $k=0 \ldots p$ :

- $\rho_{k}=2 \lambda_{k}(B) /\left(\lambda_{k}(A)+\sqrt{\lambda_{k}^{2}(A)-4 \lambda_{k}^{2}(B)}\right)$

- $\rho_{p+1+k}=2 \lambda_{k}(B) /\left(\lambda_{k}(A)-\sqrt{\lambda_{k}^{2}(A)-4 \lambda_{k}^{2}(B)}\right)$

In the case of $A=a M$ and $B=\frac{1-a}{2} M$, the computation simply comes down to $\rho_{p+1}=2 \lambda_{0}\left(\frac{1-a}{2} M\right) /\left(\lambda_{0}(a M)-\sqrt{\lambda_{0}^{2}(a M)-4 \lambda_{0}^{2}\left(\frac{1-a}{2} M\right)}\right)=2 \frac{1-a}{2}(2 a-$ $1)) /\left(a(2 a-1)-\sqrt{(a(2 a-1))^{2}-4\left(\frac{1-a}{2}(2 a-1)\right)^{2}}\right)=\frac{1-a}{a-\sqrt{2 a-1}}=\frac{a+\sqrt{2 a-1}}{1-a}$. Then, as $R=1 / \rho_{p+1}$, we have the expected result.

Remark 3.3.1. Let us examine again the four limit and classical cases of Section ??:

1) When $\mu=\frac{1}{2} \chi_{[-1,1]}, a=1 / 2$ still yields $C=\infty$ and $R=1$ : nothing can be done numerically. This case corresponds to $\mathcal{M}(x, y)=\frac{1}{4} \chi_{[-1,1]}(x) \chi_{[-1,1]}(y)$, the limit generic constant kernel with maximal $2 D$ overlap.

2) When $\mu=\chi_{[-1 / 2,1 / 2]}, a=1$ also still yields $C=1$ and $R=0$ : this optimal numerical situation will be discussed in Section ??. This case corresponds to $\mathcal{M}(x, y)=\chi_{[-1 / 2,1 / 2]}(x) \chi_{[-1 / 2,1 / 2]}(y)$, the classical limit plateau kernel with no overlap.

3) When $\mu=\operatorname{Tr} i_{[-1,1]}$, we have $a=2 / 3$, so that $C=9$ and $R=1 /(2+$ $\sqrt{3}) \simeq 0.26$ (instead of $r \simeq 0.33$ in dimension one): this situation remains numerically very good, and the interpolating functions are more localized than in $1 D$. This case will be examined in Section ??; it corresponds to $\mathcal{M}(x, y)=$ $\operatorname{Tr}_{[-1,1]}(x) \operatorname{Tr} i_{[-1,1]}(y)$, the classical square pyramid.

4) For $\mathcal{M}(x, y)=\frac{1}{4}(1+\cos (\pi x))(1+\cos (\pi y))$, corresponding to $a=3 / 4$, $C=4$ and $R=1 /(3+2 \sqrt{2}) \simeq 0.17$ (instead of $r \simeq 0.2$ in dimension one), 
we have a $2 D$ sinus-like kernel which is still better numerically.

5) We observe that $R<r$ : the speed of decrease of the matrix elements around its diagonal is faster for $N$ in $2 D$ than for $M$ in $1 D$. $(R<r \Leftrightarrow$ $2 a-1<\sqrt{2 a-1}$, which is true because $1 / 2<a<1 \Leftrightarrow 2 a-1<1$.)

Thus, the localization parameter $a$, defined in the $1 \mathrm{D}$ case, plays the same key role in the $2 D$ case. It describes the F-transform in a simple manner, as well as it governs the numerical robustness of the projection : how much matrix $N$ of the projection numerically well conditioned and interpolation functions $\mathcal{N}_{k, l}(x, y)$ have a 'small' support.

\section{Fuzzy projection in higher dimension}

The generalization to any dimension $q$ of space is immediate, following the link between formulas in dimension 1 and 2 . With the same formalism we establish, skipping all identical expressions and in the same way avoiding all proofs, the analogous results. We chose, for simplicity, the same notations as in one dimension of space, denoting in particular $x=\left(x_{1}, \ldots, x_{q}\right)$ and $k=\left(k_{1}, \ldots, k_{q}\right)$.

A fuzzy partition $\mathcal{P}=\left\{\mu_{k}, k \in[0 \ldots p+1]^{q}\right\}$ of the hypercube $[0, d]^{q}$ has the following definition and property:

Definition 4.1. (qD fuzzy partition)

Let $\mathcal{S}=\left\{\omega_{k}=k \Delta, k \in[0 \ldots p+1]^{q}\right\}$ be an uniform subdivision of $[0, d]^{q}$. Kernels $\mu_{k}$ of the partition $\mathcal{P}$ are then defined similarly by $\mu_{k}(x)=$ $\Pi_{i=1 \ldots q} \mu\left(\frac{x_{i}-\omega_{k_{i}}}{\Delta}\right)$ and are supported in the q-dimensional hypercube $\Omega_{k}=$ $\Pi_{i=1 \ldots q}\left[\omega_{k_{i}-1}, \omega_{k_{i}+1}\right]$. The generic kernel $\mu$ is supported in the unit square $[-1,1]^{q}$ and is the natural tensor product of the generic kernel $\mu$ defined in dimension 1 .

Property 4.1. ( $q D$ partition of unity)

i)

$$
\forall x \in[0, d]^{q}, \sum_{k \in[0 \ldots p+1]^{q}} \mu_{k}(x)=1
$$

ii)

$$
\forall k \in[0 \ldots p+1]^{q}, \frac{1}{\Delta^{q}} \int_{[0, d]^{q}} \mu_{k}=\frac{1}{\Delta^{q}} \int_{\Omega_{k}} \mu_{k}=\left(\int_{-1}^{1} \mu\right)^{q}=1
$$


The $q$-dimensional fuzzy transform corresponds to the operator $F$ : $L^{2}\left([0, d]^{q}\right) \mapsto\left(\mathbb{R}^{p+2}\right)^{q}$ such that:

Definition 4.2. (fuzzy transform)

$$
\forall k \in[0 \ldots p+1]^{q},(F f)_{k}:=\frac{\int_{[0, d]^{q}} f \mu_{k}}{\int_{[0, d]^{q}} \mu_{k}}=\frac{1}{\Delta^{q}}\left(f \mid \mu_{k}\right)
$$

With the scalar product $u . v=\Delta^{q} \sum_{k \in[0 \ldots p+1]^{q}} u_{k} v_{k}$ on $\left(\mathbb{R}^{p+2}\right)^{q}$ we have the same expression for the adjoint fuzzy transform $F^{*}$. Then, the $(p+2)^{q}$-dimensional sub-space $\Pi, \mathrm{F}$-projection on it, and also convergence results are defined and established identically.

Identifying $\left(\mathbb{R}^{p+2}\right)^{q}$ to $\mathbb{R}^{(p+2)^{q}}$ by the bijective map $k \mapsto k_{1}+k_{2}(p+2)+$ $k_{3}(p+2)^{2}+\cdots+k_{q}(p+2)^{q}$, components of the fuzzy transform $(F f)_{k, l}$ will be now stored in a $(p+2)^{q}$-dimensional vector. Operator $F F^{*}$ is then identified to a matrix $M$ in the canonical basis of $\mathbb{R}^{(p+2)^{q}}$, and this yields:

Theorem 4.1. (expression of $q D$ F-projection and role of a))

i) $F F^{*}$ corresponds to $a(p+2)^{q} \times(p+2)^{q}$ almost bistochastic matrix which is symmetrical block tridiagonal with symmetrical tridiagonal blocks, themselves defined in the same way by an induction process with length $q-1$, and starting at $M_{1}=M$ :

$M_{q}=\left[\begin{array}{ccccccc}a M_{q-1} & \frac{1-a}{2} M_{q-1} & & & & & \\ \frac{1-a}{2} M_{q-1} & a M_{q-1} & \frac{1-a}{2} M_{q-1} & & & & \\ & \frac{1-a}{2} M_{q-1} & a M_{q-1} & \frac{1-a}{2} M_{q-1} & & & \\ & & \cdot & \cdot & \cdot & & \\ & (0) & & \cdot & \cdot & & \\ & & & & \frac{1-a}{2} M_{q-1} & a M_{q-1} & \frac{1-a}{2} M_{q-1} \\ & & & & & \frac{1-a}{2} M_{q-1} & a M_{q-1}\end{array}\right]$

It still depends only on the $1 D$ localization parameter $a$.

ii) $F F^{*}$ is invertible and has $(p+2)^{q}$ simple eigenvalues $\lambda_{k}$ such that

$$
\lambda_{k}=\Pi_{j=1}^{q}\left(a+(1-a) \cos \frac{k_{j} \pi}{p+1}\right)
$$


verifying $0<(2 a-1)^{q} \leq \lambda_{k} \leq 1$.

iii) The associated normalized "eigenvectors" are the $v_{k}$ such that

$$
\left(v_{k}\right)_{l}=\left(\frac{2}{d}\right)^{\frac{q}{2}} \Pi_{j=1}^{q} \cos \left(\frac{l_{j} k_{j} \pi}{p+1}\right)
$$

Again, they do not depend on the generic kernel.

iv) The set of functions

$$
\mathcal{B}=\left\{f_{k}=\frac{1}{\sqrt{\lambda_{k}}} F^{*} v_{k}\right\}
$$

is an orthonormal basis of $\Pi$, so that

$$
P f=\sum_{k}\left(f \mid f_{k}\right) f_{k}
$$

v) The functions

$$
\nu_{k}(x)=\sum_{i}\left(F F^{*}\right)_{(i),(k)}^{-1} \nu_{i}(x)
$$

make $P f$ reads as the following interpolation of the fuzzy transform $F f$ :

$$
P f(x)=\sum_{k}(F f)_{k} \nu_{k}(x)
$$

We have the analogous obvious result for the condition number of $M_{q}$. Nevertheless, as shown in Theorem ??, the induction process generates a matrix $M_{q}$ whose blocks consist in a $q-1$ times repeated structure of the $1 \mathrm{D}$ case matrix $M$. Even in the 3D case, we didn't succeed in computing the speed of decrease $r$ of the interpolating functions $\nu_{k}$.

Proposition 4.1. ( $q D$ numerical robustness and role of a)

$$
c(a):=\operatorname{cond}_{2}\left(F F^{*}\right)=\frac{1}{(2 a-1)^{q}}
$$


All these results show that the overlap parameter $a=\int_{-1}^{1} \mu^{2}$, even if defined in dimension 1, plays the same central role in any dimension of space. Both for describing the F-projection as for controlling its numerical robustness. In the next section, we will see that it is also an indicator for its stability.

\section{Stability of multi-dimensional fuzzy projection and localization parameter $a$}

A question arising naturally is the following: when performing a representation of a signal by fuzzy projection with different generic kernel $\mu_{1}$ and $\mu_{2}$ can we measure the risk of deviation when choosing one or another? In other terms, can we measure the stability, say the sensitivity, of the representation, balancing from a projection $P_{1}$ to another one, $P_{2}$ ? This question is subject to interpretation because it is not really well-posed. The sole purpose of the following section is to propose tracks for this question.

\subsection{Match between projection subspaces}

Here is a way to characterize, in a general manner, the match between two finite dimensional subspaces of an Hilbert space.

Let us consider two finite subspaces $F_{1}$ and $F_{2}$ of an Hilbert space, with respective orthonormal basis $\mathcal{B}_{1}=\left\{\phi_{i}^{(1)}, i=1 \ldots n_{1}\right\}$ and $\mathcal{B}_{2}=$ $\left\{\phi_{i}^{(2)}, i=1 \ldots n_{2}\right\}$. Call $G$, the $n_{1} \times n_{2}$ Gram matrix of scalar products:

$$
G=\left[\left(\phi_{i}^{(1)} \mid \phi_{j}^{(2)}\right)\right]
$$

and its singular values $\left\{\sigma_{k}\right\}$, sorted in decreasing order.

Definition 5.1.1. (match between subspaces)

We call match between $F_{1}$ and $F_{2}$ the lowest singular value of the Gram matrix of their respective basis:

$$
\operatorname{Match}\left(F_{1}, F_{2}\right)=\min _{k}\left\{\sigma_{k}\right\} \leq 1
$$

It has been proved that singular values correspond to the cosines of 'principal angles' between the two subspaces ([?]), so that the lowest one is the cosine of the highest angle. When the latter is (at worst) $\pi / 2, F_{1}$ 
and $F_{2}$ will be considered as 'distants' in this sense, and it corresponds to Match $\left(F_{1}, F_{2}\right)=0$. On the contrary, we are interested in the situation where Match $\left(F_{1}, F_{2}\right)$ approches 1 , but for somehow distant generic kernels.

Remark 5.1.1. There are other ways to model a criterion for this match, say angle or distance. None is perfect, each depends on the nature of the problem. For example finding a cut-off in the graph of $\left\{\sigma_{k}\right\}$ will indicate an incompatibility between the two subspaces, or a loss of robustness in the model with which they are related.

Consider now, with the same notations and context as in section ??, two fuzzy transforms $F_{1}$ and $F_{2}$ with kernels $\mu^{(1)}$ and $\mu^{(2)}$, and their corresponding F-projections $P_{1}$ and $P_{2}$ on projection subspaces $\Pi_{1}$ and $\Pi_{2}$, i.e. the subspaces that are used to provide the projection of the signal: their representation by fuzzy sampling $(F f)$ followed by inverse inverse fuzzy transform: interpolation by the adjoint transform $F^{*}$. Denote $\lambda_{k}^{(i)}=a_{i}+\left(1-a_{i}\right) \cos \frac{k \pi}{p+1}$, the eigenvalues of $M_{i}=F_{i} F_{i}^{*}$, and $a_{i}=\left\|\mu^{(i)}\right\|_{2}^{2}$, for $i=1,2$. Recall finally that they are associated to the same eigenvectors $v_{k}$ (Theorem ??).

As in section ??, we define a localization parameter

$$
a_{1,2}:=\left(\mu^{(1)} \mid \mu^{(2)}\right)
$$

called the 'cross localization parameter' and that measures the overlap between the two kernels. Then, we have the following result:

Theorem 5.1.1. (match between subspaces and role of localization parameters)

The $(p+2) \times(p+2)$ Gram matrix $G$ related to $\Pi_{1}$ and $\Pi_{2}$ is diagonal, with diagonal terms

$$
G_{k, k}=\sigma_{k}=\frac{\lambda_{k}^{(1,2)}}{\sqrt{\lambda_{k}^{(1)} \lambda_{k}^{(2)}}}, k=0, \ldots, p+1
$$

where $\lambda_{k}^{(1,2)}=a_{1,2}+\left(1-a_{1,2}\right) \cos \frac{k \pi}{p+1}$ are the eigenvalues of the symmetric tridiagonal matrix $F_{1} F_{2}^{*}$ with diagonal term $a_{1,2}=\left(\mu^{(1)} \mid \mu^{(2)}\right)$ and sub or super diagonal term $\frac{a_{1,2}-1}{2}$ (similarly to the case of $M$ ).

Moreover, $\sigma_{k}$ form a decreasing sequence, so that

$$
\operatorname{Match}\left(\Pi_{1}, \Pi_{2}\right)=\sigma_{p+1}=\frac{2 a_{1,2}-1}{\sqrt{\left(2 a_{1}-1\right)\left(2 a_{2}-1\right)}}
$$


Proof. Using respective basis of $\Pi_{1}$ and $\Pi_{2}$ in Theorem ??, we compute $G_{k, l}=\left(f_{k}^{(1)} \mid f_{l}^{(2)}\right)=\left(\frac{1}{\sqrt{\lambda_{k}^{(1)}}} F_{1}^{*} v_{k} \mid \frac{1}{\sqrt{\lambda_{l}^{(2)}}} F_{2}^{*} v_{l}\right)=\frac{1}{\sqrt{\lambda_{k}^{(1)} \lambda_{l}^{(2)}}} F_{2} F_{1}^{*} v_{k} . v_{l}$. As the matrix $M_{1,2}=F_{2} F_{1}^{*}=F_{1} F_{2}^{*}$ has for elements $\left(\mu_{k}^{(1)} \mid \mu_{l}^{(2)}\right)$, it is also a symmetric tridiagonal matrix (as matrix $M$ ) with the property that its diagonal terms are $a_{1,2}$ and its sub or super diagonal terms are $\frac{a_{1,2}-1}{2}$ (as both $\mu_{k}^{(1)}$ and $\mu_{k}^{(2)}$ also define partitions of unity). Thus, its orthogonal eigenvectors are the same as $M$, and its eigenvalues reads $\lambda_{k}^{(1,2)}=a_{1,2}+\left(1-a_{1,2}\right) \cos \frac{k \pi}{p+1}$. Then, $G_{k, l}=\frac{1}{\sqrt{\lambda_{k}^{(1)} \lambda_{l}^{(2)}}} \lambda_{k}^{(1,2)} v_{k} \cdot v_{l}$ proves that $G$ is diagonal and provides the first result.

Now, we have to show that $\sigma_{k}=\frac{a_{1,2}+\left(1-a_{1,2}\right) \cos \frac{k \pi}{p+1}}{\sqrt{\left(a_{1}+\left(1-a_{1}\right) \cos \frac{k \pi}{p+1}\right)\left(a_{2}+\left(1-a_{2}\right) \cos \frac{k \pi}{p+1}\right)}}$ form a decreasing sequence. Then, as Match $\left(F_{1}, F_{2}\right):=\min _{k}\left\{\sigma_{k}\right\}=\sigma_{p+1}$, the second result will be immediate.

An equivalent condition is that the function $\varphi(x)=\frac{a_{1,2}+\left(1-a_{1,2}\right) x}{\sqrt{\left(a_{1}+\left(1-a_{1}\right) x\right)\left(a_{2}+\left(1-a_{2}\right) x\right)}}$ is increasing on $[-1,1]$. Its logarithmic derivative is $\psi(x)=\frac{1-a_{1,2}}{a_{1,2}+\left(1-a_{1,2}\right) x}-$ $\frac{1}{2}\left(\frac{1-a_{1}}{a_{1}+\left(1-a_{1}\right) x}+\frac{1-a_{2}}{a_{2}+\left(1-a_{2}\right) x}\right)$. As $a_{1,2} \leq a_{i}$ for $i=1,2$, we immediately check that $\frac{1-a_{i}}{a_{i}+\left(1-a_{i}\right) x} \leq \frac{1-a_{1,2}}{a_{1,2}+\left(1-a_{1,2}\right) x}$ for $i=1,2$. Thus $\psi(x) \geq 0$ on $[-1,1]$ and $\varphi$ is increasing, which concludes the proof.

Remark 5.1.2. (relations between localization parameters)

1) Direct computation yields the upper bounds $a_{1,2} \leq a_{1}$ and $a_{1,2} \leq a_{2}$.

2) Cauchy-Schwartz inequality provides $a_{1,2} \leq \sqrt{a_{1} a_{2}}$.

3) Note that if each $a_{i}$ verifies $1 / 2<a_{i}<1$, the 'cross localization parameter' $a_{1,2}$ can be lower than $1 / 2$. For example, we have $a_{1,2}=1 / 3$ for the choice of the overlapping step and triangular generic kernels.

Again, we see that these quantities, $a_{1}, a_{2}$, and $a_{1,2}$, play a central role in analyzing the F-projection. We have now to take advantage of formula (??) to highlight a form of stability in the representation by fuzzy projection. But choosing two parameter $a_{1}$ and $a_{2}$ is not enough for computing $a_{1,2}=\left(\mu^{(1)} \mid \mu^{(2)}\right)$, as the only relation between them is the Cauchy-Schwartz inequality $a_{1,2} \leq \sqrt{a_{1} a_{2}}$ : we have to choose first $\mu^{(1)}$ and $\mu^{(2)}$. The natural question is how to make this choice in a wide range of generic kernels that also let the different computations and interpretations possible. To do this, 
let us introduce a simple reasonable model for parametrizing kernels of fuzzy partitions.

5.2. Illustration of the stability of the F-projection with a simple model: power functions $\mu^{[\alpha]}$

Definition 5.2.1. (power generic kernels)

For $\alpha \in \mathbb{R}_{+}$we define the generic kernel $\mu^{[\alpha]}$ such that $\mu^{[\alpha]}(x)=$ $2^{\alpha-1}(x+1)^{\alpha}, \forall x \in[-1,-1 / 2]$. Then, according to definition ??, $\mu^{[\alpha]}$ is defined on $[-1,0]$ by symmetry around the point $(-1 / 2,1 / 2)$, and completed by parity on $[-1,1]$.

We give some examples of such kernels in Figure ?? for $\alpha \geq 1$.

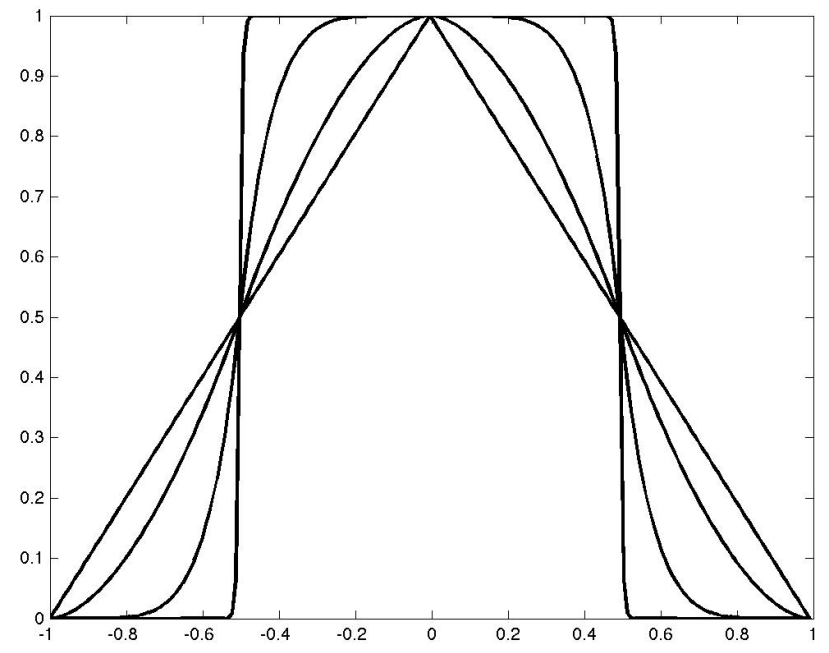

Figure 2: Examples of power generic kernels for $\alpha=1 ; 1.75 ; 6 ; 100$.

\section{Remark 5.2.1.}

1) $\mu^{[0]}$ is the overlapping step kernel: $\mu^{[0]}=\frac{1}{2} \chi_{[-1,1]}$.

2) $\mu^{[1]}$ is the triangular kernel: $\mu^{[1]}=\operatorname{Tr}_{[-1,1]}$.

3) $\mu^{[\infty]}$ is the basic step kernel: $\mu^{[\infty]}=\chi_{[-1 / 2,1 / 2]}$.

4) The sinusoidal kernel $\mu^{[\cos ]}(x)=\frac{1}{2}(1+\cos (\pi x))$ is very well approached by $\mu^{[1.75]}$, as $\left\|\mu^{[1.75]}-\mu^{[\cos ]}\right\|_{2} /\left\|\mu^{[\cos ]}\right\|_{2}=0.0077$. 
These power functions thus seem to fit in a simple and efficient way classical generic kernels. Moreover, a simple computation yields

$$
a^{[\alpha]}:=\left\|\mu^{[\alpha]}\right\|_{2}^{2}=1-\frac{1}{1+\alpha}+\frac{1}{2(1+2 \alpha)}
$$

and

$$
a^{[\alpha, \beta]}:=\left(\mu^{[\alpha]} \mid \mu^{[\beta]}\right)=1-\frac{1}{2(1+\alpha)}-\frac{1}{2(1+\beta)}+\frac{1}{2(1+\alpha+\beta)}
$$

Thus, it becomes possible to deal with

$$
\operatorname{Match}\left(\Pi_{1}, \Pi_{2}\right)=\frac{2 a_{1,2}-1}{\sqrt{\left(2 a_{1}-1\right)\left(2 a_{2}-1\right)}}
$$

using a wide class of kernels.

In Figure ??, we show the match computed this way. We can estimate that the zone indicating a good stability between two fuzzy partitions roughly starts when $a_{1}, a_{2} \geq \frac{2}{3}$. This corresponds to the triangular kernel. Thus, as those represented in Figure ??, generic kernels $\mu^{[\alpha]}$ seem to lead to a good stability for $\alpha \geq 1$ (as $a^{[1]}=2 / 3$ ). In other terms, changing the partition for the fuzzy projection does not imply an important deviation in the representation when we consider kernels near to the triangular one.

Also, we point out that the match formula is the same in any dimension of space $q$. According to equation(??), formula (??) becomes:

Proposition 5.2.1. (match in dimension q)

$$
\operatorname{Match}\left(\Pi_{1}, \Pi_{2}\right)=\sigma_{(p+1, \ldots, p+1)}=\left(\frac{2 a_{1,2}-1}{\sqrt{\left(2 a_{1}-1\right)\left(2 a_{2}-1\right)}}\right)^{q}
$$

In particular, 2-dimensional kernels that should lead to a certain stability should be near the classical square pyramidal kernel. This stands in any dimension of space, according a favor in the linear interpolation, when considering that a fuzzy overlap between cells $\Omega_{k}$ is necessary for a good description or representation of the uncertainty of the considered signal or multi-dimensional object. 


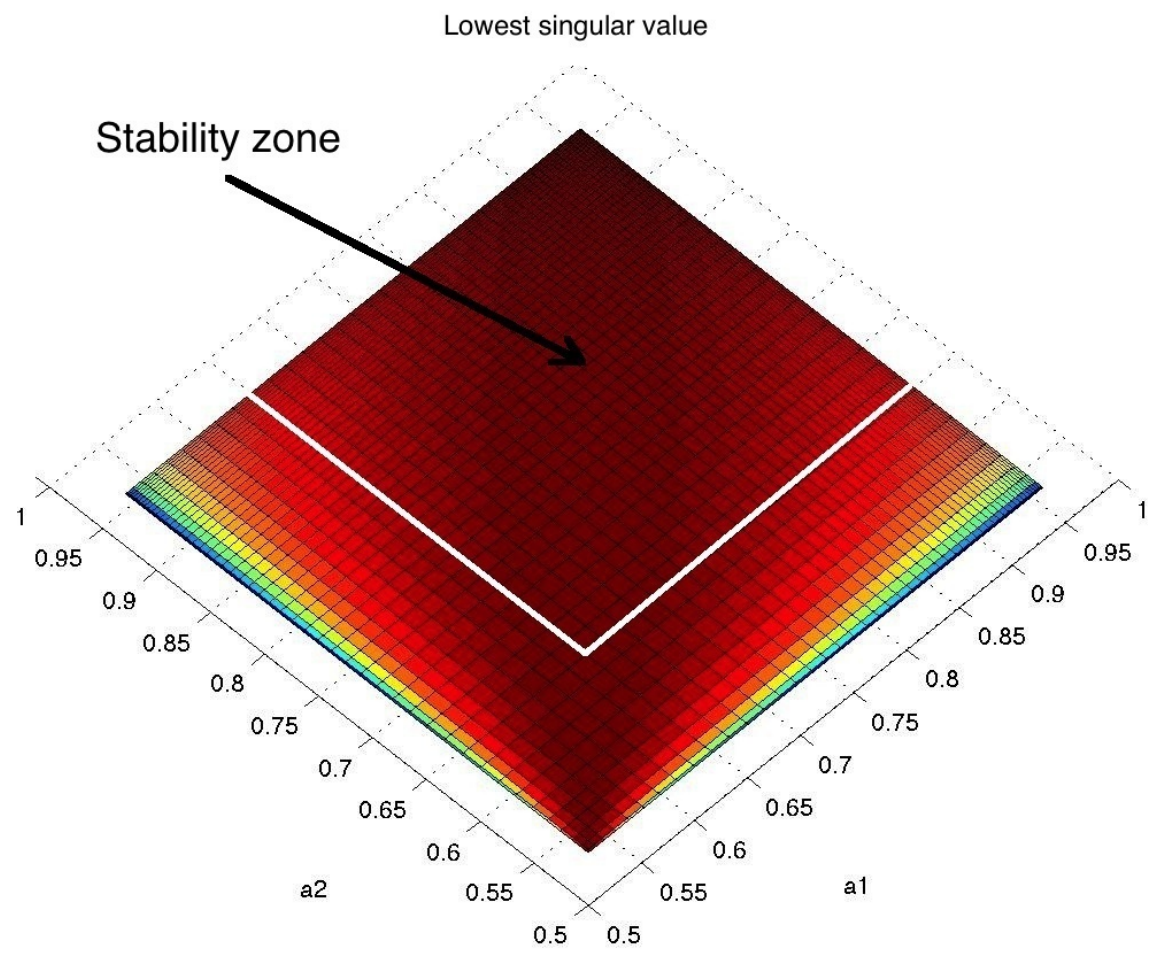

Figure 3: Match between projection subspaces according the localization parameters $a_{1}$ and $a_{2}$, using the power generic kernels $\mu^{[\alpha]}$.

\section{Conclusion}

Representation of signal and images by fuzzy and inverse fuzzy transforms has led to interesting numerical applications, as mentioned in the introduction ([?], [?], [? ], [? ], [? ], [? ], [? ]). Fuzzy projection itself, introduced in [?],[? ], is a direct simple tool directly related to these techniques, and, in addition to noise removal, has the advantage to take better into account high frequency of signals.

In this study, we have shown that multi-dimensional fuzzy projection is a very simple mathematical tool for representing multi-dimensional objects, and is analogous to the 1D case for signals, so that it should present the same numerical advantages. For instance, our next work ([? ]) will be to use the $2 \mathrm{D}$ the fuzzy projection for simultaneous denoising and enhancement 
of very noisy images, in concurrence with classical and well-tested methods. A project is also to reconstruct 3D objects, taking benefit of the easy implementation of the F-projection in any dimension.

In any case, F-projection is in practice easier to handle than a wavelet or related transform representation, because it only demands the choice of a generic kernel and its associated partition. Moreover, we have highlighted the fact that a key parameter, the localization of the generic kernel in one dimension of space, $a=\|\mu\|_{2}^{2}$, permits to illustrate and control the representation in any dimension of space. It also governs its numerical robustness (condition number of the matrix playing the central role in the projection) and its stability - say its sensitivity when modifying the sampling partition. Moreover, as studied in [? ],[? ], F-projection offers an interesting flexibility, as soon as the kernel and the diameter of the partition can be locally modified in order to better represent some regions of the signal having different behaviors (more oscillating ones for instance).

Another track for a future work is to show that, in a more precise manner, or in a more specific context of application, the linear - triangular or pyramidal - fuzzy projection is the most simple and natural one. Indeed, we have highlighted the fact that the sensitivity of the representation with respect to modifications of its generic kernel is low for the choice of the linear kernel.

For all these reasons, F-projection should be a good numerical compromise between easy implementation, numerical robustness and stable representation in the requirements of fuzzy representations.

[1] N.M. Boffi, J.C. Hill, M.G. Reuter, Characterizing the inverses of block tridiagonal, block Toeplitz matrices Computational Science $\mathscr{E}$ Discovery 8 (2015).

[2] J.-F. Crouzet, The fuzzy projection: a simple adaptive representation of signals, IEEE International Conference on Signal Processing (ICSP 2010), Beijing, China, October 2010

[3] J.-F. Crouzet, Fuzzy projection versus Inverse fuzzy transform as sampling/interpolation schemes, Fuzzy Sets and Systems 193 (2012) 108121.

[4] J.-F. Crouzet, Fuzzy projection, a tool for simultaneous compression and restoration of very noisy images, In preparation. 
[5] S. Jiang, Angles between Euclidean subspaces, Geometricae Dedicata 63, 2 (1996) 113-121.

[6] F. Di Martino, V. Loia, I. Perfilieva, S. Sessa, An image coding/decoding method based on direct and inverse fuzzy transforms, International Journal of Approximate Reasoning 48 (2008) 110-131.

[7] R. Nabben, Two-sided bounds on the inverses of diagonally dominant tridiagonal matrices, Linear Algebra and its Applications 287 (1999) 289-305

[8] G. Patanè, Fuzzy transform and least-squares approximation: Analogies, differences, and generalizations, Fuzzy Sets and Systems 180 (2011) $41-54$.

[9] R. Penrose, A generalized inverse for matrices, Proceedings of the Cambridge Philosophical Society 51 (1955) 406-413.

[10] I. Perfilieva, Fuzzy transforms, Transactions on Rough Sets II , LNCS 3135, J.F. Peters et al. Eds. (2004) 63-81.

[11] I. Perfilieva, Fuzzy transforms: theory and applications, Fuzzy Sets and Systems 157 (2006) 993-1023.

[12] I. Perfilieva, R. Valášek, Fuzzy Transforms in Removing noise, Advances in Soft Computing 2 (2005) 221-230.

[13] I. Perfilieva, Fuzzy Transforms and Their Applications to Image Compression, Lecture Notes in Computer Science (2006) 19-31.

[14] I. Perfilieva, V. Novák, A. Dvořák, Fuzzy transform in the analysis of data, International Journal of Approximate Reasoning 48 (2008) 36-46.

[15] M. Štěpnička, O. Polakovič, A neural network approach to the fuzzy transform, Fuzzy Sets and Systems 160 (2009) 1037-1047.

[16] M. Štěpnička, R. Valášek, Numerical Solution of Partial Differential Equations with Help of Fuzzy Transform, IEEE International Conference on Fuzzy Systems, 2005, pp. 1104-1109.

[17] O. Strauss, F. Graba, An interval-valued inversion of the non-additive interval-valued F-transform: use for upsampling a signal, Preprint. 
[18] J. Todd, Basic Numerical Mathematics, Birkhauser, Basel, and Academic Press, New York, 1977. 